\title{
Impact on Length of Stay of a Hospital Medicine Emergency Department Boarder Service
}

\author{
Kimiyoshi J Kobayashi, MD, MBA ${ }^{1 *}$, Steven J Knuesel, MD², Benjamin A White, MD³, Marjory A Bravard, MD², \\ Yuchiao Chang, PhD², Joshua P Metlay, MD, PhD², Ali S Raja, MD, MBA, MPH³, Melissa LP Mattison MD²
}

\begin{abstract}
${ }^{1}$ Division of General Internal Medicine, Department of Medicine, Johns Hopkins University School of Medicine, Baltimore, Maryland; ${ }^{2}$ Division of General Internal Medicine, Department of Medicine, Massachusetts General Hospital, Boston, Massachusetts; ${ }^{3}$ Department of Emergency Medicine, Massachusetts General Hospital, Boston, Massachusetts.
\end{abstract}

BACKGROUND: It is not known whether delivering inpatient care earlier to patients boarding in the emergency department (ED) by a hospitalist-led team can decrease length of stay (LOS).

OBJECTIVE: To study the association between care provided by a hospital medicine ED Boarder (EDB) service and LOS.

DESIGN, SETTING, AND PARTICIPANTS: Retrospective cross-sectional study (July 1, 2016 to June 30, 2018) conducted at a single, large, urban academic medical center. Patients admitted to general medicine services from the ED were included. EDB patients were defined as those waiting for more than two hours for an inpatient bed. Patients were categorized as covered EDB, noncovered EDB, or nonboarder.

INTERVENTION: The hospital medicine team provided continuous care to covered EDB patients waiting for an inpatient bed.

PRIMARY OUTCOME AND MEASURES: The primary outcome was median hospital LOS defined as the time period from ED arrival to hospital departure. Secondary outcomes included ED LOS and 30-day ED readmission rate.

RESULTS: There were 8,776 covered EDB, 5,866 noncovered EDB, and 2,026 nonboarder patients. The EDB service covered $59.9 \%$ of eligible patients and $62.9 \%$ of total boarding hours. Median hospital LOS was 4.76 (interquartile range [IQR] 2.90-7.22) days for nonboarders, 4.92 (IQR 3.00-8.03) days for covered EDB patients, and 5.11 (IQR 3.16-8.34) days for noncovered EDB $(P<.001)$. Median ED LOS for nonboarders was 5.6 (IOR 4.2-7.5) hours, 20.7 (IQR 15.8-24.9) hours for covered EDB, and 10.1 (IQR 7.9-13.8) hours for noncovered EDB $(P<.001)$. There was no difference in 30-day ED readmission rates.

CONCLUSION: Admitted patients who were not boarders had the shortest LOS. Among boarded patients, coverage by a hospital medicine-led EDB service was associated with a reduced hospital LOS. Journal of Hospital Medicine 2020;15:147-153. @ 2020 Society of Hospital Medicine mergency department (ED) crowding and boarding of patients awaiting admission to the hospital (ED boarding) are growing problems with important clinical care and public safety implications..$^{1-4}$ Increased ED boarding times have been associated with lower patient satisfaction, inadequate care of critically ill patients, adverse events, and increased mortality. ${ }^{3,5-7}$ Furthermore, ED boarding can diminish the ED's ability to evaluate new patients. ${ }^{5,8,9} \mathrm{ED}$ boarding is more severe in hospitals with high inpatient occupancy with resultant disproportionate burden on large urban institutions. ${ }^{24,5,10}$

Earlier studies suggest, but have not consistently shown, an association between longer ED length of stay (LOS) and

\footnotetext{
*Corresponding Author: Kimiyoshi J. Kobayashi, MD, MBA; E-mail: Kimi. kobayashi@jhmi.edu; Telephone: 410-502-9236

Published online first November 20, 2019

Find Additional Supporting Information in the online version of this article.
}

Received: July 18, 2019; Revised: September 27, 2019;

Accepted: September 27, 2019

๑) 2020 Society of Hospital Medicine DOI 10.12788/jhm.3337 longer overall hospital LOS. ${ }^{5}$ This association implies that the additional time spent in the ED waiting for a bed does not meaningfully contribute to advancing the required inpatient care. Thus, this waiting time is "dead time" that is added to the overall hospital duration.

The complexity and the volume of medical patients boarding in the ED can challenge the resources of an already overtaxed ED staff. Potential solutions to mitigate ED boarding of medicine patients generally focus on reducing barriers to timely movement of patients from the ED to an inpatient unit. ${ }^{1,3,11-13} \mathrm{UI}-$ timately, these barriers are a function of inadequate hospital capacity (eg, hospital beds, staffing) and are difficult to overcome. Two primary strategies have been used to reduce these barriers. One strategy focuses on shifting inpatient discharge times earlier to better match inpatient bed supply with ED demand. ${ }^{14-19}$ Another common strategy is utilizing inpatient attendings to triage and better match bed needs to bed availability. ${ }^{20-22}$

A separate area of interest, and the focus of this study, is the deployment of inpatient teams to hasten delivery of inpatient care to patients waiting in the ED.,23 One institution implemented an "ED hospitalist" model. ${ }^{23}$ Another creat- 


\begin{tabular}{|c|c|c|c|c|c|}
\hline & All Patients & Covered EDB & Noncovered EDB & Nonboarder & $P$ Value \\
\hline Patients, N (\%) & $16,668(100)$ & $8,776(53)$ & $5,866(35)$ & $2,026(12)$ & \\
\hline Gender, N (\%) & & & & & .38 \\
\hline Male & $9,191(55)$ & $4,826(55)$ & $3,270(56)$ & $1,095(54)$ & \\
\hline Female & $7,477(45)$ & $3,950(45)$ & $2,596(44)$ & $931(46)$ & \\
\hline Age, mean (SD) & $62.8(19.2)$ & $62.2(19.3)$ & $63.8(19.0)$ & $62.5(19.0)$ & $<.001$ \\
\hline Race, N (\%) & & & & & .14 \\
\hline White & $13,073(78)$ & $6,847(78)$ & $4,602(78)$ & $1,624(80)$ & \\
\hline African American & $1,446(9)$ & $760(9)$ & $527(9)$ & $159(8)$ & \\
\hline Asian & $533(3)$ & $285(3)$ & $184(3)$ & $64(3)$ & \\
\hline Hispanic & $175(1)$ & $84(1)$ & $70(1)$ & $21(1)$ & \\
\hline Other & $1,218(7)$ & $682(8)$ & $396(7)$ & $140(7)$ & \\
\hline N/A & $223(1)$ & $118(1)$ & $87(1)$ & $18(1)$ & \\
\hline Payor, N (\%) & & & & & $<.001$ \\
\hline Commercial & $5,364(32)$ & $2,831(32)$ & $1,843(31)$ & $690(34)$ & \\
\hline Medicare & $8,115(48)$ & $4,181(48)$ & $2,978(51)$ & $956(47)$ & \\
\hline Medicaid & $2,420(15)$ & $1,324(15)$ & $809(14)$ & $287(14)$ & \\
\hline Other & $769(5)$ & $440(5)$ & $236(4)$ & $93(5)$ & \\
\hline ED Arrival, N (\%) & & & & & $<.001$ \\
\hline $1: 00$ AM-6:59 AM & $1,729(10)$ & $846(10)$ & $759(13)$ & $124(6)$ & \\
\hline $7: 00$ AM - $12: 59$ PM & $4,769(29)$ & $1,757(20)$ & $2,456(42)$ & $556(27)$ & \\
\hline 1:00 PM -6:59 PM & $6,086(37)$ & $3,526(40)$ & $1,663(28)$ & $897(44)$ & \\
\hline 7:00 PM -12:59 AM & $4,084(25)$ & $2,647(30)$ & $988(17)$ & $449(22)$ & \\
\hline Inpatient Bed Request, N (\%) & & & & & $<.001$ \\
\hline 1:00 AM -6:59 AM & $3,201(19)$ & $2,015(23)$ & $894(15)$ & $292(14)$ & \\
\hline 7:00 AM -12:59 PM & $2,406(14)$ & $932(11)$ & $1,360(23)$ & $114(6)$ & \\
\hline 1:00 PM -6:59 PM & $5,363(32)$ & $2,067(24)$ & $2,574(44)$ & $722(36)$ & \\
\hline 7:00 PM -12:59 AM & $5,679(34)$ & $3,760(43)$ & $1,038(18)$ & $881(43)$ & \\
\hline Admission Diagnoses, $\mathrm{N}(\%)$ & & & & & $<.001$ \\
\hline Respiratory & 2,309 (14) & $1,280(15)$ & $804(14)$ & $225(11)$ & \\
\hline Circulatory & $2,169(13)$ & $1,031(12)$ & $859(15)$ & $279(14)$ & \\
\hline Digestive & $2,021(12)$ & $1,019(12)$ & $743(12)$ & $259(13)$ & \\
\hline Genitourinary & $1,184(7)$ & $658(7)$ & $389(7)$ & $141(7)$ & \\
\hline Endo & $915(5)$ & $456(5)$ & $339(6)$ & $120(6)$ & \\
\hline Mental/Behavior & $805(5)$ & $434(5)$ & $280(5)$ & $91(4)$ & \\
\hline Other conditions & $7,261(44)$ & $3,898(44)$ & $2,452(41)$ & $911(45)$ & \\
\hline Hospital Discharge Disposition, N (\%) & & & & & $<.001$ \\
\hline Home & $11,828(71)$ & $6,276(72)$ & $4,089(70)$ & $1,463(72)$ & \\
\hline Facility & $3,588(22)$ & $1,832(21)$ & $1,341(22)$ & $415(20)$ & \\
\hline AMA & $549(3)$ & $325(4)$ & $149(3)$ & $75(4)$ & \\
\hline Deceased & $384(2)$ & $180(2)$ & $162(3)$ & $42(2)$ & \\
\hline Other & $323(2)$ & $163(1)$ & $125(2)$ & $32(2)$ & \\
\hline
\end{tabular}

Abbreviations: AMA, against medical advice; ED, emergency department; EDB, emergency department boarder

ed a hospital medicine team to provide inpatient medical care to ED boarder patients and to lend clinical input to bed management. 8

At our large, urban academic medical center, the Department of Medicine in collaboration with the Department of Emergency Medicine created a full-time hospital medicine team dedicated to providing care in the ED for patients awaiting admission to a general medicine unit. We present our multiyear experience with this ED-based hospital medicine team. We hypothesized that this new team would expedite inpatient care delivery to medical boarder patients, thereby reducing the overall hospital LOS.

\section{METHODS}

\section{Study Setting and Design}

This retrospective cross-sectional study, approved by the Institutional Review Board, was conducted at a 1,011-bed academic medical center in the northeast United States. The study pe- 
TABLE 2. Throughput Metrics for EDB Patient Groups

\begin{tabular}{|c|c|c|c|}
\hline & $\begin{array}{l}\text { Covered EDB } \\
(n=8,776)\end{array}$ & $\begin{array}{l}\text { Noncovered EDB } \\
(n=5,866)\end{array}$ & $\begin{array}{c}\text { Nonboarder } \\
(n=2,026)\end{array}$ \\
\hline Median Hospital LOS, median (IQR) & $\begin{array}{l}4.92 \text { days }^{\mathrm{a}} \\
(3.00-8.03)\end{array}$ & $\begin{array}{c}5.11 \text { days } \\
(3.16-8.34)\end{array}$ & $\begin{array}{c}4.76 \text { days } \\
(2.90-7.22)\end{array}$ \\
\hline Median ED LOS, median (IQR) & $\begin{array}{l}20.7 \text { hours }^{\mathrm{a}} \\
(15.8-24.9)\end{array}$ & $\begin{array}{l}10.1 \text { hours } \\
(7.9-13.8)\end{array}$ & $\begin{array}{l}5.6 \text { hours } \\
(4.2-7.5)\end{array}$ \\
\hline 30-day Same-Institution Readmission Rate & $19.7 \%$ & $19.1 \%$ & $18.4 \%$ \\
\hline
\end{tabular}
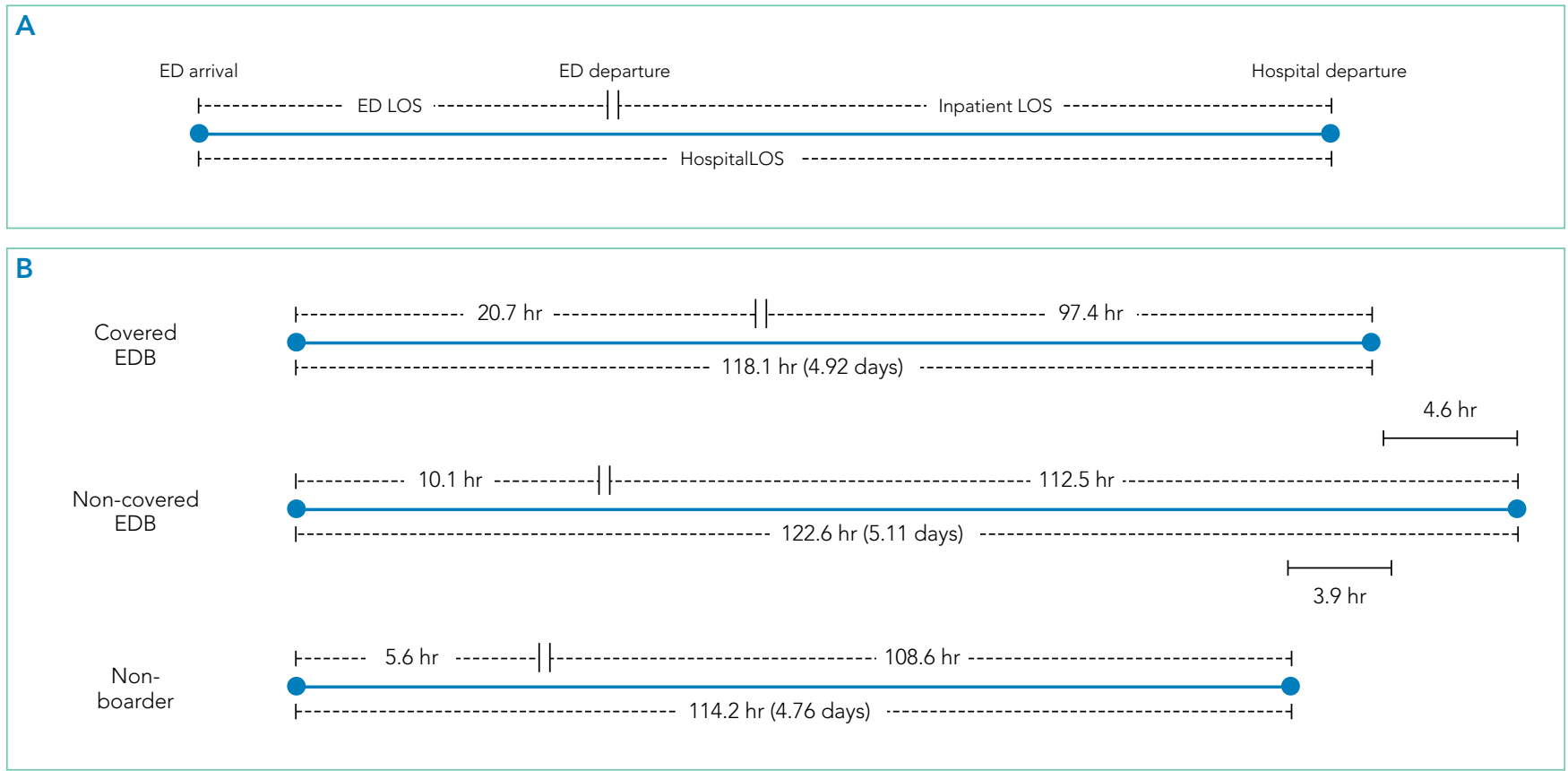

FIG. Length of Stay (LOS) Components for Emergency Department Boarder (EDB) groups. (A) Component definitions of ED LOS, inpatient LOS, and hospital LOS. (B) Median LOS values for covered EDB, noncovered EDB, and nonboarder patients. There was a 4.5 hour difference in the median hospital LOS between covered and noncovered EDB patients. There was a 3.9 hour difference in the median hospital LOS between noncovered EDB patients and nonboarders.

riod was July 1, 2016 through June 30, 2018, which was divided into Academic Year 16 (AY) (July 1, 2016 to June 30, 2017) and AY17 (July 1, 2017 to June 30, 2018).

The Hospital Medicine Unit (HMU) was a 60 full-time equivalent hospital medicine group consisting of 80 physicians and 25 advanced practice providers (APPs). During the study, the general medical services cared for an average of 260 patients per day on inpatient units with a wide variety of diagnoses and comorbidities. The ED had 48 monitored bed spaces for adult patients, as well as two dedicated ED observation units with 32 beds. The observation units are separate units within the hospital, staffed by ED clinicians, and were not included in this study. In 2016, the ED had a total of 110,741 patient visits and 13,908 patients were admitted to a medical service.

In 2010, the Department of Public Health for the state in which the medical center resides defined an ED boarder (EDB) patient as "a patient who remains in the ED two hours after the decision to admit." 24 According to this definition, any patient waiting for an inpatient bed for more than two hours after a bed request was considered as an EDB. Operationally, further distinctions were made between patients who were "eligible" for care by an internal medicine team in the ED versus those who were actually "covered". Before the intervention outlined in the current study, some care was provided by resident and hospitalist teams to eligible EDB patients from 2010 to 2015, although this was limited in scope. From July 1, 2015 to June 30,2016 , there was no coverage of medicine EDB patients.

\section{Intervention}

\section{ED Boarder Service Staffing}

On July 1, 2016, the HMU deployed a dedicated full-time team of clinicians to care for boarding patients, which was known as the EDB service. The service was created with the goal of seeing a maximum of 25 patients over 24 hours. 
Inpatient medicine attending physicians (hospitalists) and APPs worked on the EDB service. During the day (7 AM-7 PM), coverage was provided by three clinicians (generally an attending physician with two APPs). At times of increased census and demand, additional hospitalists were recruited to increase staffing on the service. During the night (7 PM-7 AM), one physician was assigned to the EDB service. When the nighttime EDB census was high, other hospitalists providing care on inpatient units were expected to help care for boarding patients in the ED. Starting July 1, 2017, the dedicated nighttime staffing for the EDB service increased to two physicians during weeknights.

There was a dedicated nursing team for the EDB service. For AY16, there were two daytime EDB nurses and one night nurse, all with a coverage ratio of three to four patients per nurse. For AY17, there were four to five daytime nurses and two to three nighttime nurses with the same coverage ratio as that for AY16. EDB nurses received special training on caring for boarder patients and followed the usual inpatient nursing protocols and assessments. During each shift, an EDB charge nurse worked in conjunction with the hospitalist, bed management, and inpatient units to determine patients requiring coverage by the EDB team.

\section{Patient Eligibility}

Similar to the workflow before the intervention, the ED team was responsible for determining a patient's need for admission to a medical service. Patients were eligible for EDB service coverage if they waited in the ED for more than two hours after the request for an inpatient bed was made. The EDB charge nurse was responsible for identifying all eligible boarder patients based on time elapsed since bed request. Patients were not eligible for the hospital medicine EDB service if they were in the ED observation units or were being admitted to the intensive care unit, cardiology service, oncology service, or any service outside of the Department of Medicine.

The EDB service did not automatically assume care of all eligible patients. Instead, eligible patients were accepted based on several factors including EDB clinician census, anticipated availability of an inpatient bed, and clinical appropriateness as deemed by the physician. If the EDB physician census was fewer than 10 patients and an eligible patient was not expected to move to an inpatient unit within the next hour, the patient was accepted by the EDB service. Patients who were not accepted by the EDB service remained under the care of the ED team until either the patient received an inpatient bed or space became available on the EDB service census. Eligible EDB patients who received an inpatient bed before being picked up by the EDB service were considered as noncovered EDB patients. Alternatively, an eligible patient may initially be declined from EDB service coverage due to, for example, a high census but later accepted when capacity allowed — this patient would be considered a covered EDB patient.

\section{Handoff and Coordination}

When an eligible patient was accepted onto the EDB service, clinical handoff between the ED and EDB teams occurred. The EDB physician wrote admission orders, including the inpatient admission order. Once on the EDB service, when space allowed, the patient was physically moved to a dedicated geographic space ( 8 beds) within the ED designed for the EDB service. When the dedicated EDB area was full, new patients would remain in their original patient bay and receive care from the EDB service. Multidisciplinary rounds with nursing, inpatient clinicians, and case management that normally occur every weekday on inpatient units were adapted to occur on the EDB service to discuss patient care needs. The duration of the patient's stay in the ED, including the time on the EDB service, was dictated by bed availability rather than by clinical discretion of the EDB clinician. When an EDB patient was assigned a ready inpatient bed, the EDB clinician immediately passed off clinical care to the inpatient medical team. There was no change in the process of assigning patients to inpatient beds during the intervention period.

\section{Study Population}

This study included patients who were admitted to the general medical services through the ED during the defined period. We excluded medicine patients who did not pass through the ED (eg, direct admissions or outside transfer) as well as patients admitted to a specialty service (cardiology, oncology) or the intensive care unit. Patients admitted to a nonmedical service were also excluded.

Two hours following a bed request, an ED patient was designated as an eligible EDB patient. Operationally, and for the purposes of this study, patients were separated into three groups: (1) an eligible EDB patient for whom the EDB service assumed care for any portion of their ED stay was considered as a "covered ED boarder," (2) an eligible EDB patient who did not have any coverage by the EDB service at any point during their ED stay was considered as a "noncovered boarder," and (3) a patient who received an inpatient bed within two hours of bed request was considered as a "nonboarder". Patients admitted to a specialty service, intensive care unit, or nonmedical services were not included in any of the abovementioned three groups.

We defined metrics to quantify the extent of EDB team coverage. First, the number of covered EDB patients was divided by all medicine boarders (covered + noncovered) to determine the percentage of medicine EDBs covered. Second, the total patient hours spent under the care of the EDB service was divided by the total boarding hours for all medicine boarders to determine the percentage of boarder hours covered.

\section{Data Sources and Collection}

The Electronic Health Record (EHR; Epic Systems Corporation, Verona, Wisconsin) captured whether patients were eligible EDBs. For covered EDB patients, the time when care was assumed by the EDB service was captured electronically. Patient demographics, admitting diagnoses, time stamps throughout the hospitalization, admission volumes, LOS, and discharge disposition were extracted from the EHR.

\section{Primary and Secondary Outcome Measures}

The primary outcome of this study was hospital LOS defined as the time from ED arrival to hospital departure (Figure). Sec- 
ondary outcomes included ED LOS (time from ED arrival to ED departure) and the rate of 30-day ED readmission to the study institution.

\section{Statistical Analysis}

SAS version 9.4 (SAS Institute, Cary, North Carolina) was used for all statistical analyses. Continuous outcomes were compared using the Mann-Whitney test and dichotomized outcomes were compared using chi-square tests. We further analyzed the differences in the primary and secondary outcomes between covered and noncovered EDB groups using a multivariable regression analysis adjusting for age, gender, race, academic year, hour of the day, and day of the week at the time of becoming an EDB. We used quantile regression and linear regression with log-transformed continuous outcomes and logistic regression for the dichotomized outcome. A $P$ value of .05 was used as a threshold for statistical significance.

\section{RESULTS}

\section{Study Population and Demographics}

There were a total of 16,668 patients admitted from the ED to the general medical services during the study period (Table 1). There were 8,776 (53\%) patients in the covered EDB group, 5,866 (35\%) patients in the noncovered EDB group, and 2,026 (12\%) patients in the nonboarder group. There were more patients admitted during AY17 compared with AY16 (8,934 vs 7,734 patients, respectively, Appendix 1). Patient demographics, including age, gender, race, insurance coverage, admitting diagnoses, and discharge disposition, were similar among all three patient groups (Table 1). A majority of patients in the covered EDB and nonboarder groups presented to the ED in the afternoon, whereas noncovered EDB patients presented more in the morning (Table 1). Consistent with this pattern, inpatient bed requests for covered EDB and nonboarder patients were more frequent between 7 PM and 7 AM, whereas bed requests for noncovered EDB patients were more frequent between 7 AM and 7 PM. Median ED volume varied by hour with a peak in volume in the afternoon hours; however, the volume of eligible and covered EDB patients had a different peak in volume around noon that was consistent across the two years (Appendix 2). Overall, $59.9 \%$ of eligible patients (excluding nonboarders) were covered by the EDB service and $62.9 \%$ of the total boarding hours were covered by the EDB service.

\section{Hospital Length of Stay}

Nonboarders had the shortest median hospital LOS (4.76; interquartile range [IQR] 2.90-7.22 days). Covered EDB patients had a median hospital LOS that was 4.6 hours ( 0.19 day) shorter compared with noncovered EDB patients (4.92 [IQR 3.008.03] days vs 5.11 [IQR 3.16-8.34 days]; Table 2). The differences among the three groups were all significant in the univariate comparison $(P<.001)$. Multivariable regression controlling for patient age, gender, race, academic year, and hour and day of the week at the time of becoming an EDB demonstrated that the difference in hospital LOS between covered and noncovered EDB patients remained significant $(P<.001)$.

\section{ED Length of Stay and 30-Day ED Readmission}

Covered EDB patients had a longer median ED LOS compared with noncovered EDB patients and nonboarder patients (20.7 [IQR 15.8-24.9] hours vs 10.1 [IOR 7.9-13.8] hours vs 5.6 [IQR 4.2-7.5] hours, respectively, Table 2). These differences remained significant in the multivariable regression models $(P<$ .001). Finally, the 30-day same-institution ED readmission rate was similar between covered and noncovered EDB patients.

\section{DISCUSSION}

We present two years of data describing a hospital medicine-led team designed to enhance the care of medical patients boarding in the ED. The period spent boarding in the $E D$ is a vulnerable time for patients, and we created the EDB service with the goal of delivering inpatient medicine-led care to ED patients awaiting their inpatient bed.

When a bed request is made in an efficient ideal world, patients could be immediately transferred to an open inpatient bed to initiate care. In our study, patients who were not EDBs (ie, waited for less than two hours for their inpatient bed) had the most time-efficient care as they had the shortest ED and hospital LOS. However, nonboarders represented only $12 \%$ of patients and the majority of patients admitted to medicine were boarders. Patients covered by the EDB service had an overall hospital LOS that was 4.6 hours shorter compared with noncovered EDB patients despite having an ED LOS that was 15.1 hours longer. These LOS differences were observed without any difference to 30-day ED readmission rates.

Given that not all boarding patients were cared by the EDB service, the role of selection bias in our study warrants discussion. Similar to other studies, ED LOS for our patient cohort is heavily influenced by the availability of inpatient beds..$^{10-12}$ The EDB service handed off patients they were covering as soon as an inpatient bed became available. Although there was discretion from the EDB charge nurse and the EDB physician about which patient to accept, this was primarily focused on choosing patients who did not have a pending inpatient bed (eg, a patient who was assigned a bed but was awaiting room cleaning). Importantly, there was no change in the bed assignment process as a part of the intervention. Our intervention's design did not allow for elucidation of causation; however, we believe that the longer ED LOS for covered EDB patients compared with noncovered EDB patients reflects the fact that the team chose patients with a higher expected ED LOS rather than that the patients had a longer LOS due to being cared by the service. Consistent with this, patients covered by the EDB service tended to have bed requests placed during the night shift compared with noncovered EDB patients; patients with bed requests at night are more likely to wait longer for their inpatient bed given that inpatient beds are generally freed up in the afternoon. We acknowledge that it is impossible to completely rule out the possibility that patient factors (eg, infectious precautions) influence inpatient bed wait time and could be another factor influencing the probability of EDB service coverage.

The current study adds to the expanding literature on EDB 
care models. Briones et al. demonstrated that an "ED hospitalist" led to increased care delivery as measured by an increased follow-up on laboratory results and medication orders. ${ }^{23}$ However, their study was not structured to demonstrate LOS changes. ${ }^{23}$ In another study, Chadaga et al. reported about their experience with a hospital medicine team providing care for EDB patients, similar to our study. ${ }^{8}$ Their hospital medicine team consisted of a hospitalist and APP deployed in the ED during the day, with night coverage provided by existing ED clinicians. They demonstrated less ED diversion, more ED discharges, and positive perceptions among the ED team. ${ }^{8} \mathrm{How}-$ ever, there was no impact on ED or hospital LOS, although their results may have been limited by the short duration of postintervention data and the lack of nighttime coverage. ${ }^{8} \mathrm{Fi}$ nally, a modeling study demonstrated a reduction in ED LOS by adding ED clinicians only for patients being discharged from the ED and not for those being admitted, although there was no explicit adjustment for LOS accounting for initiation of inpatient care in the ED. ${ }^{15}$ Extending the current literature, our study suggests that a hospitalist team providing continuous coverage to a large portion of EDB patients could shorten the overall hospital LOS for boarding patients, but even this was not enough to reduce LOS to the same level as that of patients who did not board.

Practically, there were challenges to creating the EDB service described in our study. Additional clinical staff (physician, APP, and nursing) were hired for the team, requiring a financial commitment from the institution. The new team required space within the ED footprint incurring construction costs. Before the existence of the EDB service, other ancillary services (eg, physical therapy) were unaccustomed to seeing ED patients, and thus new workflows were created. Another challenge was that internal medicine clinicians were not used to caring for patients for short durations of time before passing off clinical care to another team. This required a different approach, focusing on acute issues rather than conducting an exhaustive evaluation. Finally, the EDB service workflow introduced an additional handoff, increasing discontinuity of care. These challenges are factors to consider for institutions considering a similar EDB team and should be weighed against other interventions to alleviate ED boarding or improve throughput such as expanding inpatient capacity.

Ideal metrics to track the coverage and performance of an EDB service such as the one described in this study are undefined. It was difficult to know whether the goal should be complete coverage given the increase in handoffs, particularly for patients with short boarding times. This EDB service covered $59.9 \%$ of boarding patients and $62.9 \%$ of total boarding hours. Factors that contributed to covering less than $100 \%$ included physician staffing that was insufficient to meet demand and discretion to not accept patients expected to quickly get an inpatient bed. Therefore, the percentage of patients and boarding hours covered are crude metrics and further investigation is needed to develop optimal metrics for an EDB team.

Future studies on care models for EDB patients are warranted. Recognizing that EDB teams require additional resourc- es, studies to define which patients receive the most benefit from EDB coverage will be helpful. Moreover, the EDB team composition may need to adapt to different environments (eg, academic, urban, nonacademic, rural). Diving deeper to study whether specific patient populations benefit more than others from care by the EDB service, as measured by hospital LOS or other outcomes, would be important. Clinical outcomes, in addition to throughput metrics such as LOS, must be analyzed to understand whether factors such as increased handoffs outweigh any benefits in throughput.

There were several limitations to this study. First, it was performed at a single academic institution, potentially limiting its generalizability. However, although some workflows and team coverage structures may be institution-specific, the concept of a hospital medicine-led EDB team providing earlier inpatient care can be adapted locally and may probably achieve similar benefits. Our study population included only patients destined for general medical admission; thus, it is uncertain whether the gains demonstrated in our study would be realized for patients boarding for nonmedical services. In addition, considering the observational nature of this study, it is difficult to prove the causation that a hospitalist EDB service solely led to reductions in hospital LOS. Finally, we did not adjust for nor measure whether ED clinicians provided different care to patients whom they felt were destined for the EDB service.

In summary, nonboarder patients had the shortest overall LOS; however, among those patients who boarded, coverage by a hospitalist-led team was associated with a shorter LOS. Given the limited inpatient capacity, eliminating ED boarding is often not possible. We present a model to expedite inpatient care and allow ED clinicians to focus on newly arriving ED patients. Additional studies are required to better understand how to optimally care for patients boarding in the ED.

Disclosures: The authors have no conflicts of interest to disclose.

\section{References}

1. Asplin BR, Magid DJ, Rhodes KV, Solberg LI, Lurie N, Camargo CA, Jr. A conceptual model of emergency department crowding. Ann Emerg Med. 2003;42(2):173-180. https://doi.org/10.1067/mem.2003.302.

2. Trzeciak S, Rivers EP. Emergency department overcrowding in the United States: an emerging threat to patient safety and public health. Emerg Med J. 2003;20(5):402-405. https://doi.org/10.1136/emj.20.5.402.

3. Olshaker JS. Managing emergency department overcrowding. Emerg Med Clin North Am. 2009;27(4):593-603. https://doi.org/10.1016/jemc.2009.07.004.

4. Richardson LD, Asplin BR, Lowe RA. Emergency department crowding as a health policy issue: past development, future directions. Ann Emerg Med. 2002;40(4):388-393. https://doi.org/10.1067/mem.2002.128012.

5. Bernstein SL, Aronsky D, Duseja R, et al. The effect of emergency department crowding on clinically oriented outcomes. Acad Emerg Med. 2009;16(1):1-10. https://doi.org/10.1111/j.1553-2712.2008.00295.x.

6. Singer AJ, Thode HC, Jr., Viccellio P, Pines JM. The association between length of emergency department boarding and mortality. Acad Emerg Med. 2011;18(12):1324-1329. https://doi.org/10.1111/j.1553-2712.2011.01236.x.

7. Silvester KM, Mohammed MA, Harriman P, Girolami A, Downes TW. Timely care for frail older people referred to hospital improves efficiency and reduces mortality without the need for extra resources. Age Ageing. 2014;43(4):472-477. https://doi.org/10.1093/ageing/aft170.

8. Chadaga SR, Shockley L, Keniston A, et al. Hospitalist-led medicine emergency department team: associations with throughput, timeliness of pa- 
tient care, and satisfaction. J Hosp Med. 2012;7(7):562-566. https://doi org/10.1002/jhm.1957.

9. Lucas R, Farley H, Twanmoh J, Urumov A, Evans B, Olsen N. Measuring the opportunity loss of time spent boarding admitted patients in the emergency department: a multihospital analysis. J Healthc Manag. 2009;54(2):117-124; discussion 124-115. https://doi.org/10.1097/00115514-200903000-00009.

10. Forster AJ, Stiell I, Wells G, Lee AJ, van Walraven C. The effect of hospital occupancy on emergency department length of stay and patient disposition. Acad Emerg Med. 2003;10(2):127-133. https://doi. org/10.1111/j.1553-2712.2003.tb00029.x.

11. Hoot NR, Aronsky D. Systematic review of emergency department crowding: causes, effects, and solutions. Ann Emerg Med. 2008;52(2):126-136. https:// doi.org/10.1016/j.annemergmed.2008.03.014.

12. Asaro PV, Lewis $L M$, Boxerman SB. The impact of input and output factors on emergency department throughput. Acad Emerg Med. 2007;14(3):235-242. https://doi.org/10.1197/j.aem.2006.10.104.

13. Khare RK, Powell ES, Reinhardt G, Lucenti M. Adding more beds to the emergency department or reducing admitted patient boarding times: which has a more significant influence on emergency department congestion? Ann Emerg Med. 2009;53(5):575-585. https://doi.org/10.1016/j. annemergmed.2008.07.009.

14. Wertheimer B, Jacobs RE, Bailey M, et al. Discharge before noon: an achievable hospital goal. J Hosp Med. 2014;9(4):210-214. https://doi.org/10.1002/ jhm.2154.

15. Paul JA, Lin L. Models for improving patient throughput and waiting at hospital emergency departments. J Emerg Med. 2012;43(6):1119-1126. https:// doi.org/10.1016/j.jemermed.2012.01.063

16. Wertheimer B, Jacobs RE, Iturrate E, Bailey M, Hochman K. Discharge before noon: effect on throughput and sustainability. J Hosp Med. 2015;10(10):664669. https://doi.org/10.1002/jhm.2412.

17. Khanna S, Sier D, Boyle J, Zeitz K. Discharge timeliness and its impact on hospital crowding and emergency department flow performance. Emerg Med Australas. 2016;28(2):164-170. https://doi.org/10.1111/1742-6723.12543.

18. Patel H, Morduchowicz S, Mourad M. Using a systematic framework of interventions to improve early discharges. Jt Comm J Qual Patient Saf. 2017;43(4):189-196. https://doi.org/10.1016/j.jcjq.2016.12.003.

19. Powell ES, Khare RK, Venkatesh AK, Van Roo BD, Adams JG, Reinhardt G. The relationship between inpatient discharge timing and emergency department boarding. J Emerg Med. 2012;42(2):186-196. https://doi.org/10.1016/j. jemermed.2010.06.028.

20. Howell E, Bessman E, Kravet S, Kolodner K, Marshall R, Wright S. Active bed management by hospitalists and emergency department throughput. Ann Intern Med. 2008;149(11):804-811. https://doi.org/10.7326/0003-4819-14911-200812020-00006.

21. Howell E, Bessman E, Marshall R, Wright S. Hospitalist bed management effecting throughput from the emergency department to the intensive care unit. J Crit Care. 2010;25(2):184-189. https://doi.org/10.1016/j.jcrc.2009.08.004.

22. Howell EE, Bessman ES, Rubin HR. Hospitalists and an innovative emergency department admission process. J Gen Intern Med. 2004;19(3):266-268. https://doi.org/10.1111/j.1525-1497.2004.30431.x.

23. Briones A, Markoff $B$, Kathuria N, et al. A model of a hospitalist role in the care of admitted patients in the emergency department. J Hosp Med. 2010;5(6):360-364. https://doi.org/10.1002/jhm.636.

24. Auerbach J. Reducing emergency department patient boarding and submitting code help policies to the Department of Public Health. In: Executive Office of Health and Human Services. Boston: Department of Public Health; 2010. 